\title{
Editorial
}

\section{Jaroslaw Drelich}

Department of Materials Science and Engineering, Michigan Technological University, Houghton, MI, USA
Ludmila Boinovich

A.N. Frumkin Institute of Physical Chemistry and Electrochemistry, Russian Academy of Sciences, Russia

Ziqi Sun

Queensland University of Technology, Brisbane, Australia

In the first Original Research Article, Cao et al. ${ }^{4}$ describe the application of highly alkali-resistant disperse dyes in the alkali deweighting and dyeing of polyester textiles in a one-step process. This invention has great applied potential to simplify the technology of polyester fabric processing and reduce the fabric cleaning process. Therefore, it appears to be an environmentally sustainable and energy-saving technology for the dyeing and finishing of polyester textiles.

Nowicki ${ }^{5}$ applied the coupled map lattice (CML) method to investigate dewetting phenomenon of thin liquid films on lyophobic solid surfaces. The simulations provide useful data on both the kinetics of the dewetting process and the liquid mass distribution in the liquid film rupture product. Importantly, the CML model is capable of adequately representing key features of experimentally observed wetting behavior and is rather versatile for adjusting to different specific conditions.

An interdisciplinary and interinstitutional team of researchers from Turkey report improved bacterial anti-fouling properties of medical grade silicone surfaces that were functionalized via a plasma polymerization technique using diethyl phosphite as a precursor. $^{6}$ Bacteria anti-fouling properties were evaluated through a series of in vitro tests including nonspecific Bovine serum albumin protein adsorption, cytotoxicity studies using mouse fibroblast cells and bacterial adhesion tests using a slimeforming Staphylococcus epidermidis strain. They also conducted in vivo bacterial adhesion tests, where silicone samples with preseeded S. epidermidis bacteria were implanted into rats. All tests confirmed anti-fouling properties of the invented functionalized silicone surface against bacteria and protein.

In the last paper of this issue, Sengur-Tasdemir et al. ${ }^{7}$ from Istanbul Technical University in Turkey present a detailed study on cloning of aquaporin $\mathrm{Z}$ protein, and the preparation of nano-filtration membranes made of liposomes with aquaporin $\mathrm{Z}$ for purification of water. $^{7}$ They investigated the effect of aquaporin $Z$ addition on liposome characteristics and behavior by evaluating the adsorption performance of proteoliposomes onto membranes prepared through a spin-coating technique. The results demonstrated successful cloning of aquaporin $\mathrm{Z}$ protein from Escherichia coli $\mathrm{C} 43$ strain and its incorporation into liposomes, and concluded with membranes having water permeability increased almost five- to sixfold as compared to liposome membranes free of proteins.

We hope that everyone will enjoy reading the content of this issue. 


\section{REFERENCES}

1. Tan Q, Yin Y, Mo N, Zhang M and Atrens A (2019) Recent understanding of the oxidation and burning of magnesium alloys. Surface Innovations 7(2): 71-92, https://doi.org/10.1680/jsuin.18.00062.

2. Rad AT and Faghihi S (2019) On the relation between surface texture of metallic implants and their bioactivity. Surface Innovations 7(2): 93-100, https://doi.org/10.1680/jsuin.18.00063.

3. Bormashenko E (2019) Motion of the liquid on the surface of Leidenfrost droplets and the hairy ball theorem. Surface Innovations 7(2): 101-103, https://doi.org/10.1680/jsuin.19.00001.

4. Cao J, Meng C, Cheng X and Pan X (2019) Surface alkali deweighting and dyeing of polyester fabric by one-bath and one-step process.
Surface Innovations 7(2): 104-111, https://doi.org/10.1680/jsuin.18. 00049.

5. Nowicki W (2019) The coupled map lattice models of thin liquid film rupture. Surface Innovations 7(2): 112-121, https://doi.org/10.1680/ jsuin.18.00051.

6. Akdogan E, Demirbilek M, Sen $Y$ et al. (2019) In vitro and in vivo bacterial antifouling properties of phosphite plasma-treated silicone. Surface Innovations 7(2): 122-132, https://doi.org/10.1680/jsuin.18. 00050.

7. Sengur-Tasdemir R, Kılıç A, Tutuncu HE et al. (2019) Characterization of aquaporin Z-incorporated proteoliposomes with QCM-D. Surface Innovations 7(2): 133-142, https://doi.org/10.1680/jsuin.18.00057. 